\title{
EVALUACIÓN DE LA PRÁCTICA EDUCATIVA: UNA REVISIÓN DE SUS BASES CONCEPTUALES
}

EDUCATIONAL PRACTICE EVALUATION: A REVIEW OF THEORETICAL TENANTS

\section{Volumen 13, Número 1}

Enero - Abril

pp. 1-21

Este número se publicó el 30 de enero de 2013

Silvia Patricia Aquino Zúñiga Jesús Izquierdo Bélgibes Liliana Echalaz Álvarez

Revista indizada en REDALYC, $\underline{\text { SCIELO }}$

Revista distribuida en las bases de datos:

CATÁLOGO DE LATINDEX, IRESIE, CLASE, DIALNET, DOAJ, E-REVIST@S, SHERPA/ROMEO, QUALIS

Revista registrada en los directorios:

ULRICH'S, REDIE, RINACE, OEI, MAESTROTECA, PREAL, CLASCO 


\title{
EVALUACIÓN DE LA PRÁCTICA EDUCATIVA: UNA REVISIÓN DE SUS BASES CONCEPTUALES
}

\author{
EDUCATIONAL PRACTICE EVALUATION: A REVIEW OF THEORETICAL TENANTS
}

\author{
Silvia Patricia Aquino Zúñiga ${ }^{1}$ \\ Jesús Izquierdo ${ }^{2}$ \\ Bélgibes Liliana Echalaz Álvarez ${ }^{3}$
}

\begin{abstract}
Resumen: Abordar la evaluación del profesor, independientemente del nivel educativo en que se desempeña, implica tener claridad en dos aspectos: por una parte, desde qué posición conceptual se asume la evaluación y, por otra, qué se va a evaluar del profesor. En la literatura existente sobre la evaluación de la práctica educativa se encuentra, frecuentemente, el abordaje de estos dos conceptos de manera separada. El presente trabajo articula estos dos conceptos para tener una precisión conceptual de lo que implica el constructo de evaluación de la práctica educativa y su importancia en el proceso evaluativo. Para ello, primero, se realiza un recorrido teórico de la evaluación en su sentido más general y en el ámbito educativo. Segundo, se discuten los diferentes objetos, propósitos o finalidades y características de todo proceso de evaluación, independientemente del nivel educativo en el cual éste se lleve a cabo. Finalmente, asumiendo el paradigma conceptual de evaluación para mejorar, se discute el constructo de evaluación de la práctica educativa, haciendo énfasis en la diferencia con la evaluación de la práctica docente.
\end{abstract}

Palabras clave: EVALUACIÓN DOCENTE, PRÁCTICA DOCENTE, PRÁCTICA EDUCATIVA, PROCESO EVALUATIVO.

\begin{abstract}
Two key aspects of research on teacher performance evaluation, regardless of the teaching context where the research is to be conducted, are the theoretical foundations to support the evaluation process and the dimensions to evaluate in the teaching performance. While these two aspects should be theoretically interconnected within the research design, they are often addressed as independent aspects, both in theoretical and empirical research. This paper aims at bridging these two concepts by putting forward the construct of educational practice evaluation and its relevance to teacher evaluation. Thus, the paper first reviews the evolution of the construct of evaluation, within a general and educational framework. Then, the goal, focus, and steps of educational evaluation research are described. Finally, arguing that teacher evaluation results should primarily lead to educational quality improvement, the construct of educational practice evaluation is discussed, spotting out differences between teacher performance evaluation and educational practice evaluation.
\end{abstract}

Key words: TEACHER EVALUATION, TEACHING PRACTICE, EDUCATIONAL PRACTICE, EVALUATION PROCESS.

\footnotetext{
${ }^{1}$ Labora como profesora-investigadora en la Universidad Juárez Autónoma de Tabasco, México. Doctora en Educación por la Universidad de la Habana, Cuba. Dirección electrónica: saquinozuniga@gmail.com.mx

2 Profesor-Investigador de la Universidad Juárez Autónoma de Tabasco y Profesor Adjunto de la Universidad de Québec en Montréal, Canadá. Doctor en Enseñanza/Adquisición de Segundas Lenguas por la Universidad McGill, Canadá. Dirección electrónica: jesus.izquierdo@mail.mcgill.ca

${ }^{3}$ Asociada de Investigación en la División Académica de Educación y Artes de la Universidad Juárez Autónoma de Tabasco. Licenciada en Idiomas por la Universidad Juárez Autónoma de Tabasco. belgi90@hotmail.com
}

Artículo recibido: 29 de agosto, 2012

Aprobado: 22 de noviembre, 2012 


\section{INTRODUCCIÓN}

La evaluación, hoy más que nunca, forma parte integral en los sistemas educativos tanto en el ámbito internacional como nacional. El énfasis y propósito que adquiera dependerá del nivel del sistema educativo al que esté referido. De esta forma, el proceso de evaluación puede centrarse en la institución, en el currículum, en los programas educativos, en los profesores, en el aprendizaje, en los servicios, etc.

Antes de iniciar un proceso de evaluación, se hace necesario definir su función (el qué), y finalidad (para qué), pues es a partir de estos elementos que se definen los criterios e indicadores que guiarán el proceso evaluativo (Elola y Toranzos, 2000). Sin embargo, una de las críticas hechas a los sistemas de evaluación existentes es que, generalmente, carecen de una dimensión conceptual de la evaluación; es decir, se implementan evaluaciones sin una base conceptual que guíe el proceso, y permita definir su función y finalidad, quedándose estos sistemas en el plano técnico e instrumental (Díaz Barriga, Ángel; Barrón, Concepción, y Díaz Barriga, Frida, 2008).

El objetivo de este artículo es presentar la base conceptual de evaluación y la evaluación de la práctica educativa, con el objetivo de fundamentar un sistema de evaluación. Esta base conceptual se necesita, porque en la literatura revisada sobre evaluación se pueden encontrar tantas definiciones como teorías relacionadas con este concepto. Por ejemplo, de los estudiosos del campo que se han preocupado al respecto, se perciben distintas posiciones filosóficas, epistemológicas y metodológicas que, de acuerdo con el contexto en que surgieron, han predominado en un momento $u$ otro.

En el presente escrito, primero, se hace un recorrido conceptual de la evaluación en su sentido más general, para observar la transición del término de evaluación desde las teorías de la organización a la evaluación en el ámbito educativo. Posteriormente, se especifican los aspectos a considerar en cualquier proceso de evaluación, donde uno de los elementos fundamentales es definir el ámbito y función de la evaluación, ya que estos determinan el resto del proceso evaluativo. Seguido, contextualizando la evaluación en el ámbito educativo, se define el objeto de la evaluación; al respecto, se hace especial énfasis en la diferenciación conceptual del término de evaluación de la práctica docente versus evaluación de la práctica educativa. Finalmente, en la última sección del documento se establecen unas reflexiones finales con respecto a la importancia de la conceptualización de la evaluación educativa, sus 
bases conceptuales y su operacionalización durante el desarrollo de un sistema de evaluación.

\section{PRECISIONES CONCEPTUALES DE EVALUACIÓN}

En su sentido más general, la UNESCO (Organización de las Naciones Unidas para la Educación, la Ciencia y la Cultura, por sus siglas en inglés, 1998, p. 6) define a la evaluación como "el proceso de relevamiento y tratamiento de informaciones pertinentes, válidas y fiables para permitir a los actores interesados tomar las decisiones que se impongan para mejorar las acciones y los resultados". Por su parte, Jacques L' Ecuyer (citado por Fernández, 2005), considera que se debe evaluar para mejorar la calidad, descubriendo fortalezas y debilidades para tomar las decisiones necesarias; también, enfatiza que debe emitirse un juicio de valor sobre la institución y sus programas, fundamentado en bases sólidas con criterios y estándares conocidos y aceptados, teniendo en cuenta la misión y los objetivos institucionales.

Las diversas definiciones existentes del término evaluación se pueden agrupar dependiendo del criterio utilizado por otros autores, ya sea de acuerdo con los momentos del proceso evolutivo (Tejedor, Francisco, y García, Ana 1996), al proceso de enseñanza y de aprendizaje y al ámbito instrumental vinculado a la organización de la educación (Jornet, Mellá, y Leyva, Yolanda, 2008). No obstante, debe hacerse notar que el empleo de la evaluación desde el ámbito instrumental es el más criticado, dado que los procesos evaluativos se centran más en la aplicación de una serie de instrumentos desvinculados de un paradigma conceptual que determina el por qué y cómo de la evaluación, que en el uso de los resultados de dicha evaluación.

Con respecto a la evolución del término "evaluación", House (1993) considera que su transformación ha sido en relación con los siguientes aspectos: el conceptual, el estructural, el metodológico y el práctico.

a. El conceptual porque se identifica un amplio pluralismo (de objetos, de métodos, de criterios). El recorrido conceptual de la evaluación ha girado en torno a los atributos del objeto a evaluar, a la diferenciación entre evaluación y medición, y a los procesos sistemáticos de recogida de información. 
b. El estructural, por el reconocimiento del carácter multidisciplinar de la evaluación. Es decir, la evaluación puede abordarse desde diversos enfoques como el enfoque de la administración, de la gestión, de la educación y de la investigación.

c. El metodológico, porque se aceptan como legítimas las aproximaciones cuantitativas y cualitativas, centrando la discusión de las aproximaciones en la posible complementariedad.

d. El práctico, dado que la evaluación ha evolucionado desde la concepción instrumental hacia una visión "iluminativa”, que reconoce su valor cultural y político.

A su vez, Jiménez (1999) categoriza las definiciones de evaluación por la congruencia entre los autores con respecto a: 1) las normas o atributos de la evaluación, 2) la diferenciación entre la evaluación y la medición, 3) la toma de decisiones, y 4) la integración de los aspectos antes mencionados. Se muestra en la Tabla $N^{\circ} 1$.

Tabla № 1

Definiciones de evaluación (Jiménez, 1999, pp. 37- 42)

\begin{tabular}{|c|c|c|}
\hline Definiciones referidas a: & Autores & Característica \\
\hline $\begin{array}{l}\text { Normas o atributos de la } \\
\text { evaluación }\end{array}$ & $\begin{array}{l}\text { Tyler } \\
\text { Lafourcade } \\
\text { Mager } \\
\text { Bloom y otros }\end{array}$ & $\begin{array}{l}\text { Definiciones vinculadas con la } \\
\text { evaluación de alumnos, unas de } \\
\text { manera explícita y otras implícita. } \\
\text { Destaca la preocupación por el } \\
\text { logro de objetivos-resultados- como } \\
\text { el objeto fundamental de la } \\
\text { evaluación en un acercamiento } \\
\text { puntual de los procesos formativos. }\end{array}$ \\
\hline $\begin{array}{l}\text { Mérito o valor, diferencia entre } \\
\text { evaluación y medición. }\end{array}$ & $\begin{array}{l}\text { Scriven } \\
\text { Suchman } \\
\text { Nevo } \\
\text { Joint Comitee }\end{array}$ & $\begin{array}{l}\text { Estas definiciones van más allá que } \\
\text { la comparación con estándares y la } \\
\text { medición, donde evaluar implica la } \\
\text { emisión de un juicio de valor. }\end{array}$ \\
\hline $\begin{array}{l}\text { Orientación a la toma de } \\
\text { decisiones }\end{array}$ & $\begin{array}{l}\text { Cronbach } \\
\text { Stufflebeam y Shinkfield } \\
\text { Fernández }\end{array}$ & $\begin{array}{l}\text { En estas definiciones se incluyen } \\
\text { múltiples objetos de evaluación. } \\
\text { Este planteamiento completa a los } \\
\text { anteriores y posibilita incorporar } \\
\text { nuevas funciones y momentos } \\
\text { evaluativos y darles pleno sentido } \\
\text { (evaluación diagnóstica, evaluación } \\
\text { formativa y evaluación sumativa). } \\
\text { Se preocupa por la utilidad de la } \\
\text { evaluación en la línea de } \\
\text { perfeccionamiento o mejora de la } \\
\text { práctica, con independencia de }\end{array}$ \\
\hline
\end{tabular}




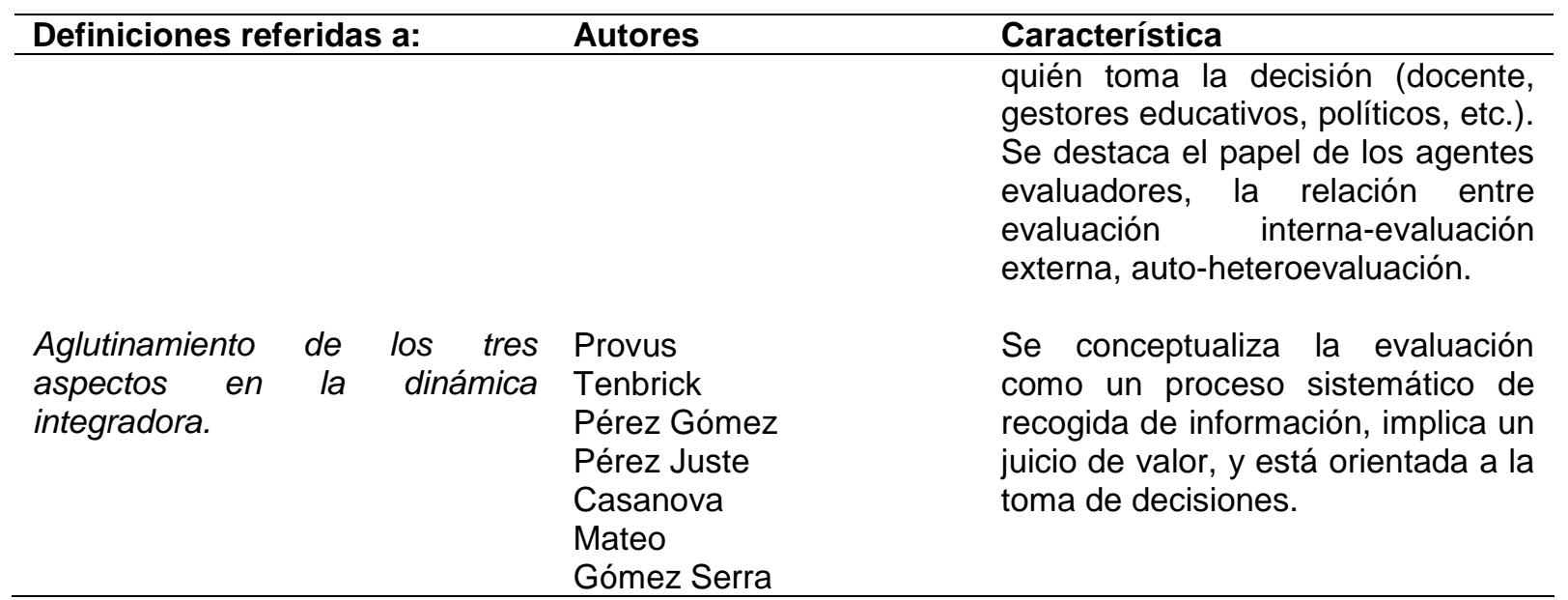

Fuente: Elaboración propia de los autores.

A diferencia de Jiménez (1999), Muñoz y Biel (2009) establecen el desarrollo del concepto de evaluación por generaciones. Como se aprecia en la Tabla № 2, en estas generaciones, el evaluador pierde su carácter de juez para convertirse en un investigador de procesos, e integra la autoevaluación y la coevaluación como elementos importantes para una cultura de evaluación para la calidad.

Tabla № 2

Desarrollo del concepto de evaluación (Muñoz y Biel, 2009, pp. 16-25)

\begin{tabular}{|c|c|}
\hline Generación & Características \\
\hline $\begin{array}{l}\text { 1ra generación o generación } \\
\text { medicional }\end{array}$ & $\begin{array}{l}\text { Vigente hasta finales de los años } 60 \text {. } \\
\text { Se fundamenta en que lo enseñado en la escuela se podía } \\
\text { evaluar utilizando los tests de memoria como medida para } \\
\text { determinar si los estudiantes dominaban los contenidos de las } \\
\text { materias. } \\
\text { Medición y evaluación eran considerados sinónimos. }\end{array}$ \\
\hline & $\begin{array}{l}\text { El papel del evaluador era de técnico experto en la aplicación } \\
\text { de instrumentos de evaluación para medir cualquier atributo. }\end{array}$ \\
\hline
\end{tabular}

2da generación

Se piensa que no solo los estudiantes deben formar parte de la evaluación educativa, siendo necesario involucrar otros elementos como el currículo, los programas, estrategias pedagógicas, materiales instruccionales, organización y otras más.

El rol del evaluador es de descriptor y medidor. 


\section{Generación}

3ra generación o generación de juicios.

\section{Características}

Se introduce el término juicio en la evaluación y el evaluador se convierte en un juez, manteniendo las cualidades de las generaciones anteriores.

Aparece la evaluación para la toma de decisiones, basada en la sociología de la organización, análisis de sistemas, teoría y práctica de la administración. 4ta generación o generación consensuada o de negociación.
Los resultados de la evaluación no son descripciones que indican cómo funcionan las cosas, sino que constituyen construcciones significativas de los actores.

La evaluación tiene una orientación hacia la toma de decisiones y el curso de las acciones a realizar se logra mediante la negociación.

El evaluador pierde la característica de juez, convirtiéndose en un investigador de procesos, capaz de construir hechos que le permitan comprender el acto de aprender y valorarlo. 5ta generación o generación de la evaluación para la Calidad
La evaluación para la calidad tiene un elemento fundamental y prioritario que es la autoevaluación, y coevaluación, elementos fundamentales para desarrollar una cultura de evaluación para la calidad.

Fuente: Elaboración propia de los autores.

Siguiendo con la descripción de Muñoz y Biel (2009), la tendencia actual de la evaluación es la de Evaluación para la Calidad. Sus bases teóricas se sustentan en la teoría general de sistemas y la teoría organizacional. Para estos autores, la evaluación para la calidad se da cuando los participantes del proceso (evaluador-evaluado) interrogan y discuten sustantivamente: ¿Qué es realmente evaluar? La evaluación para la calidad tiene un elemento fundamental y prioritario que es la autoevaluación, con la cual cada actor en su escenario, podrá valorar sus fortalezas, identificar sus debilidades, saber dónde está y hacia dónde quiere ir.

A partir de la autoevaluación, el evaluado asumirá su responsabilidad y podrá reorientar su proyecto de vida y la forma como se ha venido ejecutando. No obstante, Muñoz y Biel señalan que esta autoevaluación debe ir acompañada de una coevaluación. Esta toma un papel importante, ya que la mirada de otros permite conocer y valorar situaciones que desde nuestra óptica no son visibles, ya sea porque no queremos ver o no sabemos cómo verlas. Estos elementos son fundamentales para desarrollar una cultura de evaluación para la calidad. 
Las tendencias sobre evaluación, desde el plano conceptual de Jornet, Mellá, y Leyva, Yolanda, (2008) y Muñoz, Gabriel, y Biel, Noel (2009), coinciden en identificar el proceso evaluativo con un carácter de investigación y bases teóricas, una diversidad metodológica e instrumental, y la implicación de los actores involucrados. Estas tendencias también incluyen un enfoque de la evaluación para la mejora de la calidad y la toma de decisiones a partir de los resultados; por lo tanto, la autoevaluación y coevaluación son elementos indispensables para hacer un uso positivo de la evaluación, como lo señalan Muñoz y Biel (2009).

Una característica importante en toda evaluación es que debe estar orientada hacia la toma de decisiones (Elola y Toranzo, 2000). El proceso evaluativo ha de tener una utilidad; en este sentido, la toma de decisiones debe ir orientada a la mejora de la práctica. Esto significa, además, que la evaluación ha de ser un medio, pero no un fin en sí misma. Sin embargo, la toma de decisiones no es exclusiva de los evaluadores, otras personas ajenas a ellos, pueden tomar las decisiones, es decir, la función principal del evaluador está en la valoración, pero no necesariamente en la toma de decisiones, que puede corresponder a los responsables del programa o de los objetivos a evaluar.

Como puede observarse, en las diversas definiciones sobre evaluación, hay una diversidad de objetos de referencia cuyo proceso evaluativo es similar. Cada definición corresponde a una situación dada, es decir, la evaluación pasa de ser considerada como sinónimo de medición y de emisión de un juicio de valor, a un elemento esencial para la toma de decisiones, mediante la negociación. En cualquier caso, el proceso de evaluación presenta planteamientos multivariados en cuanto a los instrumentos, las técnicas y los métodos, así como de los agentes involucrados en ella.

A partir de este recorrido conceptual, podemos entonces definir la evaluación como un proceso sistemático de recogida de información, no improvisado, y necesitado de organizar sus elementos. Además, en este proceso, se hace necesario sistematizar sus fases, temporalizar sus secuencias, proveer los recursos, y construir o seleccionar los instrumentos. También, implica un juicio de valor, lo que significa que no basta con recoger ordenadamente la información, que servirá para establecer las metas, estrategias y acciones para realimentar a los participantes en el proceso de evaluación y así contribuir a la mejora. En virtud del auge que ha tenido la evaluación en el contexto educativo, además de efectuar su precisión conceptual, es importante, en las secciones que siguen, destacar su rol y describir su proceso en el ámbito educativo. 


\section{3.- EVALUACIÓN Y SU PROCESO EN EL ÁMBITO EDUCATIVO}

El inicio del siglo XXI, sin lugar a dudas, se ha caracterizado por el énfasis de la evaluación en el ámbito educativo, tanto en el plano internacional como nacional. Esta evaluación es el resultado de políticas educativas internacionales y nacionales que demandan a las instituciones y sistemas educativos de todos los niveles, emplear la evaluación como un mecanismo de mejora. Estas exigencias conllevan, de este modo, a la extrapolación del concepto de evaluación, en su sentido más general, al ámbito educativo, y hacen necesario definir "la evaluación educativa" y su objeto de evaluación.

Según Valenzuela (2008, p.16), la evaluación educativa se define como un proceso, y a la vez como un producto. Su aplicación nos permite estimar el grado en el que un proceso educativo favorece el logro de las metas para las que fue creado. Este autor explica, también, que en el ámbito educativo pueden considerarse como objetos de la evaluación: la institución, el currículum, los programas, el aprendizaje, y los maestros. Por su parte, Ramos (citado por Muñoz y Biel, 2008, p. 35), define la evaluación educativa como el proceso sistemático de búsqueda de información sobre una realidad educativa para conocerla y comprenderla, y así poder emitir un juicio de valor sobre ella. Este juicio de valor está orientado hacia la toma de decisiones y a la mejora de dicha realidad. Para este autor, evaluar es un proceso científico que tiene las siguientes características:

- Orienta la toma de decisiones, así como la mejora de la calidad del proceso y del producto.

- Dirige todas las unidades posibles de análisis e involucra a los diferentes actores de la educación.

- Asume diversidad metodológica.

- Identifica a la medición como componente del proceso, y

- Supone instrumentos de medida y de valores.

Asumiendo una postura integradora de la evaluación, Jornet y Leyva (2008), destacan en la evaluación educativa, los diferentes aspectos:

- Identificación de un proceso evaluativo con carácter de investigación aplicada y de características propias.

- Diversidad de objetos o unidades evaluadas.

- Diversidad metodológica en búsqueda de la complementariedad. 
- Integración de diferentes objetivos que conllevan la emisión de un juicio de valor, la orientación de la toma de decisiones, y finalidades formativas y sumativas.

- La calidad como eje central de referencia.

- La reflexión sobre los métodos para identificar y construir indicadores y criterios que operativicen la idea de calidad, reconociendo el papel de la teoría, los expertos y las audiencias.

- Integración de la medición como componente del proceso evaluativo, vinculado a la recogida de datos y análisis de la información.

- La utilización positiva de la evaluación, comprometida con la mejora de la situación evaluada, y valorada desde los criterios de utilidad y credibilidad.

La evaluación, como se ha visto, se considera un proceso sistémico, (vinculado a la teoría general de sistemas); y sistemático (que procede de una forma organizada), de relevamiento de información que permite elaborar juicios acerca de cierto objeto, en comparación con un conjunto de criterios elaborados para una finalidad determinada. Ruiz (1998, p. 7), en el campo de lo educativo, entiende el proceso de la evaluación como: “...proceso de análisis estructurado y reflexivo, que permite comprender la naturaleza del objeto de estudio y emitir juicios de valor sobre el mismo, proporcionando información para ayudar a mejorar y ajustar la acción”. Por su parte, Elola y Toranzos (2000), establecen que el proceso de evaluación debe contemplar las siguientes fases.

La fase de planificación permite articular todos los factores que intervienen en el proceso de evaluación con la intencionalidad de garantizar la veracidad y rigor de los datos, así como la validez y eficacia de las conclusiones. También, permite organizar los diferentes elementos, sistematizar las fases de desarrollo e identificar los recursos necesarios. Debe contemplar:

a. Descripción clara del problema, objeto o ámbito de evaluación. Establecer claramente qué es lo que se va a evaluar: aprendizajes, instituciones, sistema educativo, programas o proyectos, profesores.

b. Función de la evaluación. Que puede ser simbólica, política, de conocimiento, de mejoramiento, de desarrollo de capacidades o contractual.

c. Elección del enfoque metodológico apropiado. 
d. Especificación de los indicadores, criterios, preparación de los procedimientos y/ o instrumentos de recogida de datos. Incluye la asignación de responsabilidades a involucrados, la temporalización de las diferentes fases y actuaciones, el presupuesto económico.

e. Análisis e interpretación de los datos. Referida a la previsión de los mecanismos de discusión y elaboración de conclusiones,

f. Informe. Establecer claramente el contenido, la forma y a quién estará dirigido: a directores, profesores, estudiantes, padres de familia.

La fase de ejecución tiene como objetivo principal recoger la información necesaria sobre la que sustenta los juicios de valor. Del mayor o menor rigor con que se ejecuten todos sus pasos dependerá la fiabilidad y velocidad de la información y consecuentemente la validez de las conclusiones.

La fase de análisis de datos tiene como propósito el tratamiento de los datos recolectados, mediante procedimientos racionales y estadísticos. Se trata de sintetizar lo obtenido para dar respuesta a los propósitos de la evaluación. En relación con el fin y la naturaleza de los datos, se abren nuevas decisiones vinculadas al almacenamiento de la información recolectada, los criterios que guían el análisis y el tipo de análisis en sí mismo, junto a la revisión de las preguntas centrales que formula el valor de la evaluación.

El análisis de datos puede darse de manera cuantitativa, a partir de análisis estadísticos como son la frecuencia, comparaciones, tendencias. Los datos cualitativos pueden tratarse empleando análisis de contenido, narrativa, seguidos análisis inferenciales que resulten de la contrastación o triangulación de instrumentos y actores involucrados en el proceso.

Un aspecto importante al iniciar un proceso de evaluación es tener claridad con respecto a su función y finalidad. Tal pareciera que función y finalidad significan lo mismo. De acuerdo con el diccionario de la Real Academia de la Lengua Española (2000, p. 87), función significa tarea que corresponde realizar a una institución o entidad o a sus órganos o personas. Finalidad se define como fin con qué o por qué se hace algo (p. 56).

En la revisión de la literatura, encontramos autores que no establecen esta diferencia y solo se enfocan a la finalidad de la evaluación. Por ejemplo, la finalidad de la evaluación puede usarse para: la medición del logro de objetivos (Tyler, 1950); la toma de decisiones a 
partir de los resultados (Stufflebeam, 1967; Alkin, 1969; Cronbach, 1982); la valoración de respuesta a las necesidades de los participantes, (Stake, 1967; Scriven, 1973); la interpretación y comprensión del objeto evaluado (Parlett y Hamilton, 1977). Casanova (1992), por su parte, define la evaluación por su finalidad o función, en evaluación formativa (por poner el énfasis en el valor educativo y formador de las situaciones de evaluación) y evaluación sumativa (por establecer balances fiables de los resultados obtenidos al final de un proceso de enseñanza y de aprendizaje).

No obstante, en el presente trabajo se parte de las postura de Elola y Toranzos (2000, pp. 7-9), ya que estas autoras vinculan y definen claramente las funciones y finalidades de la evaluación aplicada a diferentes ámbitos u objetos en el contexto educativo.

\section{Funciones}

- Simbólica. La evaluación está asociada con el cumplimiento de un proceso evaluativo, sin que necesariamente haya un impacto.

- Política. La evaluación es de carácter instrumental para la toma de decisiones.

- De conocimiento. La evaluación implica el incremento del conocimiento y comprensión del objeto evaluado, donde la retroalimentación y dar cuenta de los resultados son dos formas de esta función.

- De mejoramiento. La evaluación implica la toma de decisiones, complementada por la función del conocimiento y política.

- De desarrollo de capacidades. La evaluación contribuye a incrementar el desarrollo de dispositivos técnicos instrumentales valiosos y poco estimulados.

- Contractual. La evaluación se relaciona con la claridad en los criterios de evaluación de los aprendizajes dentro del aula.

\section{Finalidades}

- De diagnóstico. Enfatiza los componentes vinculados con la producción sistemática de la información.

- De pronóstico. Enfatiza el valor productivo que pueda tener la información que se genera.

- De selección. Enfatiza la utilización de la información con propósitos de selección. 
- De acreditación. Enfatiza el valor social simbólico que tiene la evaluación y las consecuencias que los resultados tienen para el individuo o institución.

Una característica que debe cumplir un proceso de evaluación es que sea manifiesta; es decir, que las personas involucradas, profesores, directores, estudiantes, conozcan la finalidad y función, y que sea confidencial. De tal forma que los agentes implicados no vean la evaluación como una amenaza, sino como una oportunidad de crecer; con resultados compartidos; tender a la autoevaluación y, en lo posible, que sea un proceso sistemático y sistémico.

Cuando se implementa un proceso de evaluación educativa, independientemente del nivel en que se desarrolle, es importante tener claridad conceptual de la evaluación y de su proceso. En la fase de planeación, dependiendo de la postura conceptual que se asuma, se definirá el ámbito u objeto de evaluación, la función y las finalidades de la evaluación. A partir de estas definiciones, se determinará la elección del enfoque metodológico, la especificación de los indicadores, y el uso que se le dará al análisis e interpretación de los resultados.

Estos aspectos deben quedar claramente manifestados para los actores involucrados, para que la evaluación se vea como una oportunidad de crecimiento y no como una amenaza.

\section{4.- eVAluación de la pRÁctica docente Y DE LA PRÁctica EDUCATIVA}

Una parte esencial del proceso de evaluación la constituye, sin lugar a dudas, el ámbito u objeto de evaluación. Como indica Valenzuela (2008, p.16), en el ámbito educativo pueden considerarse como objetos de la evaluación: la institución, el currículum, los programas, el aprendizaje, y los profesores. Cuando la evaluación se centra en catedráticos, es necesario definir los aspectos de éste que serán evaluados. En este sentido, pueden evaluarse, por ejemplo, su formación, desempeño académico, y desempeño docente. En el presente trabajo, nos enfocaremos en la evaluación del desempeño docente; este concepto vincula la práctica docente y la práctica educativa, términos que parecieran sinónimos, pero que, conceptualmente, tienen sus diferencias, alcances y limitaciones.

En relación con estos dos conceptos, en el ámbito de la educación superior, Rueda y Díaz-Barriga (2004) establecen una diferencia conceptual entre evaluación académica y evaluación de los docentes. La primera considera una gama de actividades que las 
instituciones del nivel superior demandan de "los académicos": la docencia, la tutoría y asesoría; la difusión de la cultura, la elaboración de materiales didácticos y la investigación.

La evaluación "de los docentes," sin embargo, está referida a las actividades vinculadas, de forma directa, con el proceso de enseñanza y de aprendizaje; lo que el pedagogo realiza antes, durante y después de que ocurre un episodio didáctico con su pensamiento, tanto explícito como implícito; con la actividad magisterial; con la práctica profesional como enseñante, así como la reflexión sobre esta. Esta diferenciación está vinculada con las actividades que se le demandan a un académico universitario y que necesariamente responden a un marco curricular del nivel superior.

Con respecto a la evaluación docente, todas estas actividades corresponden al proceso educativo, donde Slavin (2002), nos dice que para evaluar el proceso educativo desde el aula, independientemente del nivel educativo que se trate, debemos considerar los siguientes elementos: a) calidad de instrucción, la cual se refiere al modo en que se presenta la información o destrezas a los estudiantes para que las aprendan fácilmente; b) nivel apropiado de instrucción, que se relaciona con el grado de habilidad del maestro para asegurarse de que todos los educandos estén preparados para aprender una lección; c) los incentivos relacionados con el grado en que el profesor se cerciora de que los educandos estén motivados para trabajar en los ejercicios y para aprender el nuevo material; y d) asignación de tiempo por tareas, que se refiere a la necesidad de dar tiempo suficiente a los alumnos para que aprendan el nuevo material. Sin embargo, al interior de la evaluación del docente, con respecto a los elementos antes mencionados, hay una diferenciación conceptual entre práctica docente, la cual se desarrolla en el aula independientemente del contexto institucional o curricular del pedagogo, y la práctica educativa, enmarcada por el contexto institucional. (De Lella, Cayetano, 2011; Slavin, Ernest, 2002; García, Benilde, y Navarro, Francisco, 2008a).

La práctica docente es concebida como la acción que el profesor desarrolla en el aula, especialmente referida al proceso de enseñar (De Lella, 2011), y es multidimensional, debido a los diversos acontecimientos simultáneos que en ella ocurren (Doyle, 1986 citado por García et al, 2008a). La práctica docente responde a las interrogantes; cómo lo hace, con qué lo hace, para qué lo hace. Bajo la perspectiva de Tejedor y García (1996), algunos de los puntos a evaluar en la práctica docente son: las actividades instructivas o aquellas referentes al salón de clases; la actividad investigadora del docente y la utilidad que dichas 
investigaciones tienen; el compromiso con el departamento de la asignatura a impartir; y por último, el compromiso con la comunidad a la que pertenece y su entorno de trabajo.

La práctica educativa, por su parte, se concibe como el conjunto de situaciones dentro del aula, que configuran el quehacer del profesor y del estudiante, pero que responden a un marco curricular e institucional, con determinados objetivos de formación, circunscritos al conjunto de actuaciones que inciden directamente sobre el aprendizaje de los estudiantes García, et al., 2008b, p.3). El análisis de la práctica educativa debe considerarse como una actividad dinámica, reflexiva de la intervención pedagógica ocurrida antes y después de los procesos interactivos en el aula (Zabala, 2002), donde la interactividad incluye lo sucedido en el contexto del salón de clases entre el profesor, los educandos y el contenido (Coll y Solé, 2002).

Aunado a lo anterior, Arbesú y Piña (2004), García y Navarro (2001), García y Espíndola, (2004), y Reyes (2004) coinciden en reconocer que el estudio de la práctica educativa y su evaluación entrañan un reto formidable, donde se requiere construir y plantear un conjunto de ejes que puedan configurar la evaluación de dicha práctica. Evaluar la práctica educativa implica, necesariamente, incluir la valoración de la práctica docente, pues lo que el profesor realiza en el aula debe estar, necesariamente, enmarcado en un contexto curricular, que determina los objetivos y contenidos de un programa educativo; por consiguiente, desde de la práctica educativa, el docente debe dominar el conocimiento de su programa educativo.

Con respecto al conocimiento del programa educativo, Shulman (citado por GarcíaCabreo, 2008b), afirma que los maestros requieren dominar dos tipos de conocimiento: 1) el conocimiento del contenido y 2) el conocimiento pedagógico. El primero, se refiere al teóricopráctico de la asignatura; el segundo, por su parte, se relaciona con el conocimiento y creencias que tiene el maestro sobre la enseñanza y el aprendizaje, sin referirse a un dominio específico. Schulman indica que de la interacción de los dos tipos de conocimiento surge el conocimiento del contenido pedagógico de la asignatura. Este se refiere a las formas específicas de enseñar una asignatura en particular. El dominio de este tipo de contenido comprende: a) la concepción global de la docencia de una asignatura; b) el conocimiento y las estrategias sobre la instrucción; c) conocimiento de las interpretaciones, el pensamiento y el aprendizaje de los estudiantes de una asignatura; y d) el conocimiento del currículo y los materiales curriculares. 
En lo referente al conocimiento pedagógico, Danielson (2000) y Sandín (2005), lo describen como dominios, elementos o aspectos a evaluar. Para Danielson (2000), el docente debe cumplir con cuatro dominios: Planificación y Preparación, Ambiente en el Salón de Clase, Metodología de Enseñanza, Responsabilidades de Profesión. El primer dominio se refiere al conocimiento del contenido a enseñar, conocimiento con el que cuentan los estudiantes y la metodología de enseñanza y evaluación que utiliza. El segundo dominio, referido al ambiente de clase, se relaciona con la habilidad del profesor para crear un entorno propicio de aprendizaje, incluyendo aspectos físicos e interpersonales. El tercer dominio, se relaciona con las habilidades del docente para propiciar el aprendizaje en los estudiantes, incluyendo los métodos de enseñanza, las formas de corregir al discente y las técnicas para preguntar o comentar los temas de clase. Por último, el dominio de las responsabilidades de profesión, se refiere a la autoevaluación del maestro, su contribución a la institución y su profesionalismo.

El conocimiento disciplinar hace referencia a una disciplina, que de acuerdo con Sandin, (2005, p. 35), "...es el cuerpo organizado de conocimientos sobre un conjunto de cosas o acontecimientos, tales como hechos, datos, observaciones, sensaciones y percepciones, que constituyen elementos básicos del conocimiento o definiciones que delimitan las fronteras de su dominio". Este conocimiento disciplinar proporciona al profesor el conocimiento de los contenidos, que se apoya en dos bases: la bibliografía y los estudios acumulados en cada una de las disciplinas, y el saber académico histórico y filosófico sobre la naturaleza del conocimiento en estos campos de estudio. Además, este conocimiento permite al maestro definir los contenidos de la asignatura, y organizar, de manera lógicopedagógica, la dosificación y secuencia de los contenidos que han de ser enseñados y aprendidos en un determinado periodo de tiempo.

En este concepto de práctica educativa, el currículo en el cual ocurre el proceso de enseñanza y de aprendizaje, tiene un papel esencial, ya que el currículum delimita la postura teórica y práctica sobre la concepción de enseñanza y de aprendizaje, los objetivos curriculares, los tipos de contenidos y enfoques de evaluación del aprendizaje, así como la vinculación entre las diferentes asignaturas que contribuyen en la formación del perfil de egreso de un programa educativo. De esta forma, el conocer el marco curricular en que se desarrollará la práctica educativa, ayuda al profesor a entender el propósito, los programas y la organización administrativa del sistema escolar (Daramola, 1987). 
El currículo es el punto de referencia y los objetivos curriculares no sólo se refieren a los temas de contenido, sino que contiene un criterio relativo al nivel de aprendizaje requerido por los estudiantes. Los objetivos curriculares forman el pilar central de la enseñanza de una unidad o módulo, expresan lo que se quiere que comprendan los alumnos después de haberles enseñado una unidad o módulo. (Biggs, 2010). He aquí la vinculación entre el currículo y la práctica educativa, pues el currículo se convierte en el marco referencial y punto de partida para lograr una alineación entre objetivos curriculares, objetivos de la asignatura, contenidos, estrategias de enseñanza y de aprendizaje y la forma de evaluar esos aprendizajes. Este concepto de la práctica educativa permite asumir una postura teórica donde el proceso de evaluación posibilita identificar los aspectos comunes que se toman en cuenta al evaluar la práctica educativa, es decir, el conocimiento que todo profesor debe tener sobre su disciplina, y el conocimiento sobre cómo enseñarla, sin olvidar que esta práctica se da dentro de un marco curricular institucional que el maestro también debe conocer.

Empleando los referentes presentados anteriormente, en este trabajo, asumimos la evaluación de la práctica educativa como un proceso sistemático de búsqueda de información, para comprender y mejorar las acciones realizadas por el profesor. La comprensión y la mejora se darán a partir de la autoevaluación-reflexión de sí mismo, la coevaluación y la toma de decisiones. La evaluación se enfoca, entonces, en el contenido pedagógico de la asignatura antes, durante, y después de las situaciones desarrolladas por el maestro dentro del aula para incidir en el aprendizaje de los estudiantes, dentro del marco de los objetivos curriculares de un nivel educativo.

La evaluación de la práctica educativa, entonces, involucra necesariamente la práctica docente e incluye el trinomio de los aspectos esenciales, anteriormente mencionados: el currículo, el conocimiento disciplinar, y el conocimiento pedagógico. Además, a partir de este concepto de práctica educativa, la evaluación conlleva implícita la mejora de calidad y la toma de decisiones a partir de los resultados. Este concepto está ligado al propuesto por Danielson (2000), Slavin (2002) y Tejedor y García (1996), donde los dominios, elementos o aspectos a evaluar del docente tienen en común valorar aspectos vinculados al conocimiento pedagógico y aquellos aspectos relacionados con la institución. Esta concepción de la evaluación de la práctica educativa permite definir el ámbito, la función y la finalidad de la fase de planeación de todo proceso evaluativo, proporcionando, de esta manera, una 
dimensión conceptual al sistema de evaluación que se desarrolle, rebasando, de esta forma, el plano técnico instrumental en que muchos sistemas evaluativos se quedan, donde la evaluación se convierte en el fin.

\section{Reflexiones finales}

La mejora para alcanzar la calidad se ve como un slogan en los diferentes discursos y las estrategias políticas educativas. El recorrido realizado en la presente publicación sobre la transformación conceptual de la evaluación permitió analizar los propósitos, los alcances, las metodologías utilizadas, las limitaciones, los ámbitos y la complejidad para su definición.

La tendencia conceptual vigente de la evaluación para la mejora es vista como un proceso, cuyas características son propiciar la reflexión, la autoevaluación y la co-evaluación, con la finalidad de que los resultados tengan un uso positivo y no punitivo; los actores a evaluar deben tener claridad de los aspectos y utilidad de la evaluación; de esta forma, se facilita el proceso evaluativo y se evita la resistencia y su rechazo.

Conceptualmente, el término evaluación desde esta fundamentación se ha aplicado al ámbito educativo: la institución, el currículum, los programas, los estudiantes el aprendizaje, los profesores pueden ser evaluados. El análisis del recorrido conceptual y el proceso evaluativo permite percatarse de la importancia de la claridad sobre estos aspectos al implementar un proceso evaluativo, de tal forma que toda evaluación que se lleve a cabo, esté respaldada de manera conceptual, para que no se quede tan solo en el plano instrumental, como se ha criticado arduamente.

Toda evaluación necesariamente tiene un proceso que contempla las fases de planeación, ejecución, y análisis de resultados. La fase de planeación es donde se determina el ámbito a evaluar, la función y finalidad que tendrá, el enfoque metodológico que se usará, la especificación de indicadores y criterios a utilizar, el análisis de los resultados y, finalmente, el informe del proceso. (Elola y Toranzos, 2000). Sin embargo, no puede desarrollarse un proceso de evaluación sin asumir una postura teórico-conceptual de la misma. Es en la fase de planeación, donde se establece el ámbito a evaluar, que en el caso educativo, puede referirse a la institución escolar, al sistema educativo, a programas o proyectos, a los aprendizajes, y a los profesores.

En este trabajo, nos enfocamos a dos aspectos de la fase de planeación de todo proceso evaluativo: la definición del ámbito a evaluar, que en nuestro caso es la práctica 
educativa; la función de la evaluación, que es de mejoramiento y de desarrollo de capacidades, cuya función será el diagnóstico para la toma de decisiones (Elola y Toranzos, 2000). La definición de estos aspectos determina y guía el resto del proceso evaluativo, la elección del enfoque metodológico, la especificación de los indicadores, y el uso que se le dará al análisis e interpretación de los resultados.

A partir de lo anterior, es que se podrán lograr cambios significativos en los aprendizajes, si tanto profesores y alumnos logran valorar la importancia de la promoción y facilitación de esos aprendizajes, evaluados de forma cualitativa y cuantitativamente.

En la actualidad, la evaluación juega un papel protagónico en toda institución educativa, y en lo que respecta a la evaluación de estudiantes y de docentes, no debe ser vista como un acto punitivo sino como una oportunidad de cambio, de una verdadera mejora y transformación a partir de conocer y analizar la práctica educativa, para lograr cambios en los procesos de enseñanza y aprendizaje, y obtener el desarrollo integral de los estudiantes.

Precisamente, porque encontramos una diversidad de situaciones educativas en un solo país, estado, institución e incluso en el aula, no puede olvidarse en la labor interpretativa la influencia del contexto, los programas, la organización escolar, el currículum y las intenciones educativas contenidas en él, los cuales responden a un contexto social determinado.

El constructo de evaluación de la práctica educativa, asumido en este trabajo, incluye el trinomio de los siguientes aspectos esenciales: el currículo, el conocimiento disciplinar, y el conocimiento pedagógico que todo docente debe dominar, sin importar el nivel educativo en que desarrolle su práctica. Una evaluación para mejorar la calidad solo se logra, por una parte, a través de la autorreflexión y crítica de quien es evaluado y, por otra, de las decisiones que se tomen a partir de los resultados, siempre tendiente al desarrollo de las capacidades, cuando se trata de la evaluación de la práctica educativa, de lo contrario, la evaluación no tiene sentido y solo se queda en el plano técnico-instrumental.

La evaluación adquiere, entonces, una función pedagógica y didáctica de carácter integrador, dado que hace posible la integración de la unidad enseñanza, aprendizaje y evaluación enmarcados en el currículum institucional. 


\section{Referencias}

Alkin, Marvin. (1969). Evaluation theory development. En Milbrey Wallin Mc Laughlin y Denis Charles Philips, Evaluation and education at quarter century (pp. 91-112). Chicago: University of Chicago.

Arbesú, Isabel y Piña, Juan. (2004). Evaluación de la docencia desde la visión de los estudiantes: una experiencia interpretativa. En Mario Rueda Beltrán, ¿Es posible evaluar la docencia en la universidad? Experiencias en México, Canadá, Francia, España y Brasil (pp. 225-240). México: UABJO-ANUIES.

Biggs, John. (2010). Calidad del aprendizaje universitario. México: ANUIES-SEP-Narcea.

Casanova, María. (1992). La evaluación: garantía de calidad para el centro educativo. España: Edelvives.

Coll, César y Solé, Isabel. (2002). Enseñar y aprender en el contexto del aula. En César Coll, Javier Palacios y Ángel Marchesi (Coord), Desarrollo psicológico y educación 2. Psicología de la educación escolar (pp. 357-286). Madrid: Alianza.

Cronbach, Lee. (1982). Designing evaluations of educational and social programs. Chicago: Jossey-Bass.

Danielson, Charlotte y McGreal, Thomas. (2000). Teacher evaluation to enhance professional practice. Princeton: NJ: Educational Testing Service.

Daramola, S. L. (1987, enero). Student teaching in Nigeria teacher training colleges: Issues and prospects. Nigerian Education, 47-58.

De Lella, Cayetano. (2011, enero). Modelos y tendencias de la formación docente. Organización de Estados Iberoamericanos. Recuperado de http://www.oei.es/cayetano.htm

Díaz Barriga, Ángel, Barrón, Concepción y Díaz-Barriga, Frida. (2008). Impacto de la evaluación en la educación superior mexicana. México: UNAM-ANUIES-Plaza y Valdéz.

Elola, Nydia y Toranzos, Lilia. (2000). Evaluación educativa: una aproximación conceptual. Recuperado http://www.oei.es/calidad2/luis2.pdf

Elola, Nydia. (2001). Evaluación y aprendizajes. Aportes para el debate curricular. Buenos Aires: GCABSAS, Secretaría de Educación.

Fernández, Norberto. (2005). Los procesos de evaluación y acreditación universitaria en América Latina en la experiencia del MERCOSUR en la evaluación de la calidad y acreditación. Madrid: IESALC.ANECA.

García Cabrero, Benilde y Espíndola, Susana. (2004). Las contribuciones de la investigación sobre la práctica educativa. En Mario Rueda Beltrán, ¿Es posible evaluar la docencia 
en la universidad? Experiencias en México, Canadá, Francia, España y Brasil (pp.251-262). México: UABJO-ANUIES.

García Cabrero, Benilde y Navarro, Francisco. (2001). La construcción de una metodología para el análisis de la práctica educativa y sus implicaciones en la evaluación docente. En Mario Rueda, Evaluar para comprender y mejorar la docencia en educación superior (pp. 193-208). México: CESU-UNAM.

García Cabrero, Benilde; Loredo, Javier y Carranza, Guadalupe. (2008a). Análisis de la práctica educativa de los docentes: pensamiento, interacción y reflexión. Revista Electrónica de Investigación Educativa, (Especial). Recuperado de http://redie.uabc.mx/index.php/redie/article/view/200

García Cabrero, Benilde; Loredo, Javier; Carranza, Guadalupe; Figueroa, Antonio; Arbezú, Isabel; Monrroy, Mario y Reyes, Raúl. (2008b). Las aproximaciones teóricometodológicas en los trabajos de la RIED: consideraciones en torno a la construcción de un modelo de evaluación de la docencia universitaria. En Mario Rueda Beltrán, La evaluación de los profesores como recurso para mejorar su práctica (pp. 1-26). México: UNAM-Plaza y Valdéz.

House, Ernest. (1993). Professional evaluation: social impact and political consequences. CA: Sange: Newbury Park.

Jiménez, Bonifacio. (1999). Evaluación de programas, centros y profesores. España: Síntesis.

Jornet, Mellá. y Leyva, Yolanda. (2008). Conceptos, metodología y profesionalización educativa. México: INITE.

Moreno, María. (1995). Didáctica: Fundamentación y Práctica. México: Editorial Progreso.

Muñoz, Gabriel. y Biel, Noel. (2008). La quinta generación: evaluación para la calidad. Venezuela: CEINEDUCA.

Organización Mundial para la Educación, la Ciencia, Cultura y las Artes. (1998). Declaración Mundial sobre la Educación Superior en el S. XXI: Visión y Acción. Conferencia mundial sobre la Educación Superior. París: UNESCO. Recuperado de http://www.unesco.org/new/es/unesco/.

Parlett, Malcom. Y Hamilton, David. (1977). Evaluation as illumination: a new approach to the study of innovative programmes. En Hamilton D. y otros. (Eds), Beyond the numbers game. London: MacMillan.

Real Academia de la Lengua Española. (2000). Diccionario Ilustrado. México: Real Academia de la Lengua Española. P. 234 
Reyes, Raúl. (2004). Evaluación de la docencia en el nivel superior del estado de Tlaxcala. En Mario Rueda, ¿Es posible evaluar la docencia en la universidad? Experiencias en México, Canadá, Francia, España y Brasil (pp. 195-208). México: UABJO-ANUIES.

Rueda, Mario. y Díaz-Barriga, Frida. (2004). La evaluación de la docencia en la universidad. Perspectivas desde la investigación profesional. México: Plaza y Valdéz.

Ruiz, José María. (1998). Cómo hacer evaluación en centros educativos. Madrid: Narcea.

Sandin, María. (Septiembre de 2005). Retos actuales de la formación en investigación cualitativa en educación. Conferencia de clausura del I Encuentro Internacional de Investigación Cualitativa en Educación. Barquisimeto, Venezuela: UPEL Universidad Pedagógica Experimental Libertador-Instituto de Pedagogía.

Scriven, Michael. (1973). Goal-free evaluation. En E.House (Ed). School evaluation: The politicas and process (pp. 319-328). Berkely, CA: McCutchan.

Slavin, Ernest. (2002). Evidence-based education policies: transforming educational practice and research. American Educational Research Association, 15-21.

Stake, Robert. (1967). The countenance of educational evaluation. Illinois: Teacher College Record.

Stufflebeam, Daniel. (1967). Evaluative research. Principles and practice in public service and social action programs. New York: Russell Sage Foundation.

Tyler, Ralph. (1950). Basic principles of curriculum and instruction. Chicago: University of Chicago Press.

Tejedor, Franciso y García, Ana. (1996). La evaluación de la calidad de la docencia universitaria en el marco de la evaluación institucional, desde la perspectiva del alumno. En Javier Tejedor. y José Luis Rodríguez Diéguez (Coord.), Evaluación Educativa. II Evaluación Institucional. Fundamentos teóricos y aplicaciones prácticas (pp. 93-122). Salamanca: Instituto Universitario de Ciencias de la Educación.

Valenzuela, Jaime. (2008). Evaluación de instituciones educativas. México: Trillas.

Zabala, Antoni. (2002). La práctica educativa, cómo enseñar. Barcelona: Grao. 